\title{
Why Black lives matter in science
}

\author{
Systemic racism has held back under-represented minorities, especially Black people, in scientific research. We're \\ committed in our roles as journal editors to help correct this inequality.
}

A $\mathrm{t}$ the time of writing (mid-June), millions of people are still reeling from the shocking video of George Floyd, a Black man who lost his life at the hands of a police officer in Minneapolis - followed closely by another death in Atlanta of a Black man named Rayshard Brooks. Such events have amplified the sad fact that anti-Black racism remains a major issue in the United States and throughout the world. The protests that followed these deaths, propelled by the Black Lives Matter movement, are waking many people up to the injustices that Black people suffer - injustices that reach far beyond their treatment by the police.

The scientific community is not immune to such injustices: Black researchers have been held back by pervasive, systemic racism. This spurred a call for academic and STEM professionals (using the Twitter hashtags \#Strike4BlackLives, \#ShutDownSTEM and \#ShutDownAcademia) to strike on 10 June 2020, to reflect, self-educate, and come up with concrete action plans to address the problem of systemic racism in the scientific community.

"In the wake of the most recent murders of Black people in the US, it is clear that white and other non-Black people have to step up and do the work to eradicate anti-Black racism," wrote the organizers at http://www.shutdownstem.com. "Those of us who are not Black, particularly those of us who are white, play a key role in perpetuating systemic racism. Direct actions are needed to stop this injustice. Unless you engage directly with eliminating racism, you are perpetuating it." The message is clear: it is not enough to simply be not racist; one must work harder to be anti-racist.

Instead of going about our normal business on 10 June, Nature Methods editors used this day to reflect and to brainstorm what actions we as journal editors can take to help combat overt and systematic racism in the scientific community. We quickly realized that one day is hardly enough time to truly understand the depth of this issue or to put plans immediately into action. It is just the start to what we know will be a long process that will require continual attention and that will challenge us in multiple ways.

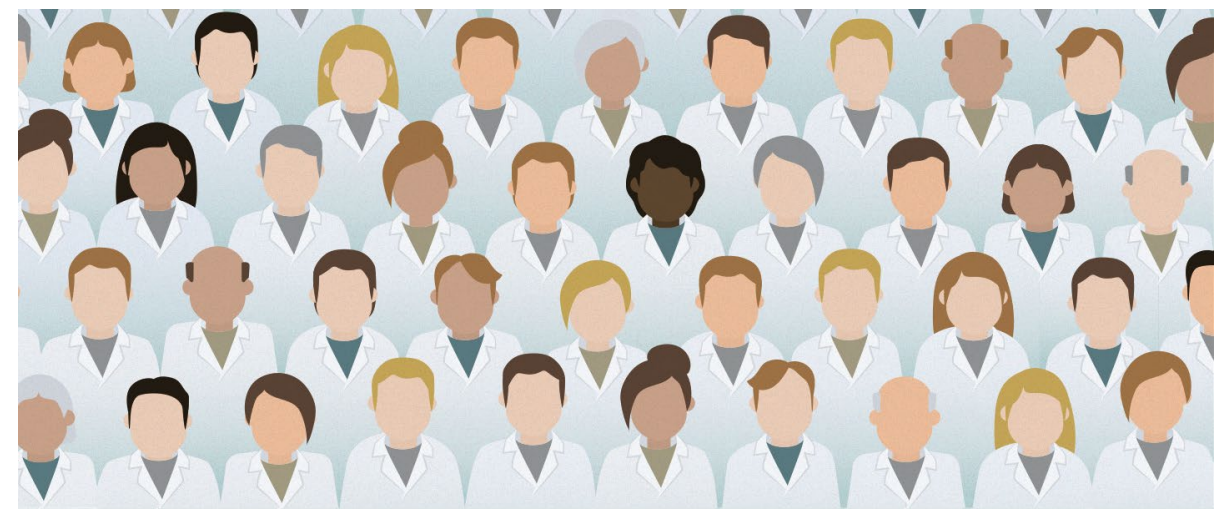

Credit: Erin Dewalt

As curators of Nature Methods, we aim to publish the best, most cutting-edge science. Most papers we publish come from well-funded labs in wealthy countries - labs that, despite some increasing diversity, still tend to be overwhelmingly run by white, male principal investigators. And so we recognize that we are complicit in a biased system that favors certain groups of people to the detriment of others. We pledge to work harder and smarter as editors to right this inequity. We commit to carefully appraising our internal decision-making processes for implicit biases.

While we have already been making a concerted effort to promote diversity in our pages, we know we can do better. We will carefully consider inclusion when inviting researchers to contribute reviews or opinion pieces. We will feature more Black scientists and other under-represented minorities in our magazine content. We will increase the diversity of our reviewer pool to include broader geographical areas. We will do more outreach, including giving talks about publishing and about editorial careers, to a more geographically diverse audience, and reach out to younger students at early career stages. Finally, we will not tolerate or engage with any researchers, no matter how famous or important, who make racist comments in our presence, whether against someone on our team or anyone else. We will hold ourselves accountable to these action plans by revisiting how we've done a year from now.
Our editorial team is diverse, but includes no Black editors. Most of us have experienced discrimination of some kind, but we do not have first-hand knowledge of the sorts of horrific experiences many Black scientists have been sharing on Twitter under the hashtag \#BlackintheIvory. We stand in solidarity with Black scientists and are committed to doing what we can to chip away at the mountain of inequality in the scientific community. With open minds, we invite our readers of any color, via e-mail or Twitter, to share ideas about journal content that might be of utility and interest, and about how we can make further positive changes.

The challenge of overturning the systemic racism ingrained in scientific culture might seem insurmountable. But there is something that everyone can do to support our Black colleagues and the broader Black Lives Matter movement, whether it is making a statement by protesting or striking, donating money to causes that promote racial justice, volunteering time or expertise to reach out to under-represented groups, putting diversity in the forefront of any selection process, reflecting on and checking one's own implicit biases, or simply speaking out when one sees racism happening.

Published online: 2 July 2020 https://doi.org/10.1038/s41592-020-0908-7 